\title{
Differences in Characteristics and Comorbidity of Cluster Headache According to the Presence of Migraine
}

\author{
Tae-Jin Song ${ }^{a *}$, Mi Ji Lee ${ }^{b *}$ \\ Yun-Ju Choi ${ }^{\mathrm{c}}$, Byung-Kun Kim ${ }^{\mathrm{d}}$ \\ Pil-Wook Chung ${ }^{e}$, Jung-Wook Park ${ }^{f}$ \\ Min Kyung $\mathrm{Chu}^{\mathrm{g}}$, Byung-Su Kim ${ }^{\mathrm{h}}$ \\ Jong-Hee Sohn', Kyungmi Oh ${ }^{\mathrm{j}}$ \\ Daeyoung Kim ${ }^{\mathrm{k}}$, Jae-Moon Kim ${ }^{\mathrm{k}}$ \\ Soo-Kyoung Kim', Kwang-Yeol Park ${ }^{m}$ \\ Jae Myun Chung ${ }^{n}$, Heui-Soo Moon ${ }^{e}$ \\ Chin-Sang Chung ${ }^{\mathrm{b}}$, Jin-Young Ahn ${ }^{\circ}$ \\ Soo-Jin Cho ${ }^{p}$ \\ aDepartment of Neurology, Ewha Womans \\ University College of Medicine, Seoul, Korea \\ bDepartment of Neurology, Neuroscience Center, \\ Samsung Medical Center, Sungkyunkwan \\ Samsung Medical Center, Sungkyunkwan \\ University School of Medicine, Seoul, Korea
'Department of Neurology, Presbyterian Medical \\ Center, Jeonju, Korea
dDepartment of Neurology, Eulji Hospital, Eulji \\ University, Seoul, Korea \\ 'Department of Neurology, Kangbuk \\ Samsung Hospital, Sungkyunkwan University \\ School of Medicine, Seoul, Korea \\ 'Department of Neurology, Uijeongbu St. Mary's \\ Hospital, The Catholic University of Korea, \\ Uijeongbu, Korea \\ 'Department of Neurology, Severance Hospital, \\ Seoul, Korea \\ hepartment of Neurology, Bundang Jesaeng \\ General Hospital, Daejin Medical Center, \\ Seongnam, Korea \\ iDepartment of Neurology, Chuncheon Sacred \\ Heart Hospital, Hallym University Collo \\ Heart Hospital, Hallym University College of \\ Medicine, Chuncheon, Korea \\ Department of Neurology, Korea University \\ College of Medicine, Seoul, Korea \\ Department of Neurology, Chungnam National \\ University College of Medicine, Daejeon, Korea \\ 'Department of Neurology, Gyeongsang Nation \\ University College of Medicine, Jinju, Korea \\ mepartment of Neurology, \\ Chung-Ang University Hospital, Seoul, Korea \\ nepartment of Neurology, Inje University \\ College of Medicine, Seoul, Korea \\ 'Department of Neurology, Seoul Medical Center, \\ Seoul, Korea \\ Department of Neurology, Dongtan Sacred \\ Heart Hospital, Hallym University College of \\ Medicine, Hwaseong, Korea
}

Received December 26, 2018

Revised January 25, 2019

Accepted January 30, 2019

\section{Correspondence}

Soo-Jin Cho, MD, $\mathrm{PhD}$

Department of Neurology, Dongtan Sacred

Heart Hospital, Hallym University College of

Medicine, 7 Keunjaebong-gil, Hwaseong

18450, Korea

Tel +82-31-8086-2310

Fax +82-31-8086-2317

E-mail dowonc@naver.com

Jin-Young Ahn, MD, PhD

Department of Neurology, Seoul Medical

Center, 156 Sinnae-ro, Jungnang-gu,

Seoul 02053, Korea

Tel $+82-2-2276-8670$

Fax +82-2-562-9883

E-mailnneuroahn@naver.com

*These authors contributed equally to this work.
Background and Purpose Cluster headache $(\mathrm{CH})$ can present with migrainous symptoms such as nausea, photophobia, and phonophobia. In addition, an overlap between $\mathrm{CH}$ and migraine has been reported. This study aimed to determine the differences in the characteristics of $\mathrm{CH}$ according to the presence of comorbid migraine.

Methods This study was performed using data from a prospective multicenter registry study of $\mathrm{CH}$ involving 16 headache clinics. $\mathrm{CH}$ and migraine were diagnosed by headache specialists at each hospital based on third edition of the International Classification of Headache Disorders (ICHD-3). We interviewed patients with comorbid migraine to obtain detailed information about migraine. The characteristics and psychological comorbidities of $\mathrm{CH}$ were compared between patients with and without comorbid migraine.

Results Thirty (15.6\%) of 192 patients with $\mathrm{CH}$ had comorbid migraine, comprising 18 with migraine without aura, 1 with migraine with aura, 3 with chronic migraine, and 8 with probable migraine. Compared to patients with $\mathrm{CH}$ without migraine, patients with $\mathrm{CH}$ with comorbid migraine had a shorter duration of $\mathrm{CH}$ after the first episode [5.4 \pm 7.4 vs. $9.0 \pm 8.2$ years (mean \pm standard deviation), $p=0.008$ ], a lower frequency of episodic $\mathrm{CH}(50.0 \%$ vs. $73.5 \%, p=$ $0.010)$, and a higher frequency of chronic $\mathrm{CH}(13.3 \%$ vs. $3.7 \%, p=0.033)$. Psychiatric comorbidities did not differ between patients with and without comorbid migraine. The headaches experienced by patients could be distinguished based on their trigeminal autonomic symptoms, pulsating character, severity, and pain location.

Conclusions Distinct characteristics of $\mathrm{CH}$ remained unchanged in patients with comorbid migraine with the exception of an increased frequency of chronic $\mathrm{CH}$. The most appropriate management of $\mathrm{CH}$ requires clinicians to check the history of preceding migraine, particularly in cases of chronic $\mathrm{CH}$.

Key Words chronic cluster headache, cluster headache, comorbidity, migraine, treatment.

\section{INTRODUCTION}

Cluster headache $(\mathrm{CH})$ is a rare primary headache disorder with very severe pain characterized by autonomic symptoms and periodicity, and is the most common type of headache categorized under trigeminal autonomic cephalagia. ${ }^{1}$ Most patients with $\mathrm{CH}$ are classified as having episodic $\mathrm{CH}$ since they have a headache-free period of several months or even years, the so-called remission period. Those patients without such a long remission period are classified as having chronic $\mathrm{CH} \cdot{ }^{1-3}$

An overlap between $\mathrm{CH}$ and migraine has been reported. In addition to autonomic symptoms, 25-46\% of patients with $\mathrm{CH}$ experience and report migraine-like features such as nausea, photophobia, phonophobia, and sometimes migraine itself. ${ }^{4-6}$ Furthermore, $\mathrm{CH}$

(a) This is an Open Access article distributed under the terms of the Creative Commons Attribution Non-Commercial License (https://creativecommons.org/licenses/by-nc/4.0) which permits unrestricted non-commercial use, distribution, and reproduction in any medium, provided the original work is properly cited. 
and migraine may share common clinical features such as unilateral pain location, similar triggering factors (histamine, calcitonin-gene-related peptide, or nitrate), a triptan response, and the treatment effect of neuromodulation. ${ }^{7-9}$ Moreover, a possible genetic link between $\mathrm{CH}$ and migraine has also been reported. ${ }^{10}$ Underlying migraine can delay the diagnosis of $\mathrm{CH}$, which is of concern since the main abortive and preventive medications differ for the two disorders. Thus, both accurately diagnosing $\mathrm{CH}$ and distinguishing $\mathrm{CH}$ from migraine are essential to the correct management of $\mathrm{CH}$. In addition, comorbidity of $\mathrm{CH}$ and migraine may reveal common neurobiological mechanisms.

A few studies have investigated the coincidence of $\mathrm{CH}$ and migraine, with comorbid migraine reportedly being present in $10-16.7 \%$ of patients with $\mathrm{CH}^{4,11,12}$ However, the differences between the characteristics of $\mathrm{CH}$ and migraine have not been evaluated adequately. Several other aspects also remain unknown, such as the times of onset of $\mathrm{CH}$ and migraine, clinical parameters for differentiation, and treatment pattern for migraine in patients who suffer from both migraine and $\mathrm{CH}$. In this study we aimed to determine the differences in the characteristics and comorbidities of $\mathrm{CH}$ according to the history of preceding migraine, and to elucidate the detailed history of migraine in patients with $\mathrm{CH}$.

\section{METHODS}

\section{Subjects}

The Korean Cluster Headache Registry (KCHR) is a prospective, multicenter, observational, registry-based study that included patients with $\mathrm{CH}$ at 16 hospitals in Korea between September 2016 and July 2018. ${ }^{13}$ In the present study, we included adult patients who were diagnosed as having $\mathrm{CH}$ (episodic, chronic, or probable $\mathrm{CH}$ ) based on the judgment of the neurology board members. $\mathrm{CH}$ was diagnosed based on the third edition of the International Classification of Headache Disorders, 3rd edition beta version (ICHD-3 beta). ${ }^{14}$ Patients who were unable to communicate in Korean, who were unable to give reliable answers to questionnaires due to psychiatric reasons or cognitive decline at the time of the investigator's decision, or who were included in other clinical studies were not registered in the KCHR. ${ }^{13,15}$ For our substudy we enrolled only those $\mathrm{KCHR}$ participants with $\mathrm{CH}$ compatible with the ICHD-3. ${ }^{1}$ Our study was approved by an Internal Review Board and a local ethics committee at each hospital (2016396-I). All patients enrolled in the KCHR had already provided written informed consent, and all procedures complied with the tenets of the Declaration of Helsinki and Good Clinical Practice guidelines.

\section{Details of the KCHR}

We have previously reported on the detailed protocol of the KCHR. ${ }^{13,16}$ The clinical data collected about the last ictal period included sex, age at onset, body mass index, time to diagnosis of $\mathrm{CH}$ from the first headache onset, $\mathrm{CH}$ severity on a numeric rating scale (from 0 to 10 ), frequency of $\mathrm{CH}$, mean duration of $\mathrm{CH}$ since the first episode, bout duration of $\mathrm{CH}$ during the ictal period, total number of $\mathrm{CH}$ bouts, smoking history (current smoker, ex-smoker, or never smoked), alcohol intake, headache impact (Headache Impact Test-6; HIT-6), anxiety (General Anxiety Disorder-7 scale; GAD-7), depression (Patient Health Questionnaire-9; PHQ-9), stress (Short-Form Perceived Stress Scale-4; PSS-4), quality of life (EuroQol: EQ-5D health utility score), and cutaneous allodynia (Allodynia Symptoms Scale-12). ${ }^{17-20}$ The bout duration was defined as the duration between the first day to the last day of cluster periods in which cluster attacks occurred in series lasting for weeks or months separated by remission periods, which usually lasted several months or even years. ${ }^{1}$ The total number of bouts was defined as total number of developing bout (cluster period) diagnosed as the $\mathrm{CH}$. For all participants the investigators ascertained the presence of comorbid migraine based on the ICHD-3. The type of migraine was classified into migraine without aura, migraine with aura, chronic migraine, and probable migraine. ${ }^{1}$

\section{Migraine substudy}

We performed a comprehensive face-to-face or telephonic interview with each patient who agreed to participate to obtain further information about migraine and its relationship with $\mathrm{CH}$. Furthermore, we assessed time parameters for diagnosing $\mathrm{CH}$ and migraine, clinical parameters for differentiating between patients with $\mathrm{CH}$ with and without migraine, and curative and preventive medications for migraine.

\section{Statistical analysis}

IBM SPSS Statistics software (version 20.0 for Windows, IBM Corporation, Armonk, NY, USA) was used for statistical analysis. Kolmogorov-Smirnov tests were performed to investigate whether the included variables conformed to a normal distribution. The chi-square test and Fisher's exact test were used to compare categorical variables, and the independent $t$-test was used to compare continuous variables. When normality was not present, continuous variables were analyzed using the Mann-Whitney U test or Kruskal-Wallis test. For convenience, the presence of migraine was dichotomized into with and without migraine regardless of the migraine classification. A probability value of $p<0.05$ in two-tailed tests was considered statistically significant. 


\section{RESULTS}

\section{Comparison between $\mathrm{CH}$ with and without comorbid migraine}

Among 194 patients identified as having $\mathrm{CH}$ based on ICHD-3, 1 patient was excluded due to not meeting ICHD-3 criteria and another patient was excluded due to an uncertain history of migraine. We therefore finally analyzed $192 \mathrm{CH}$ patients, comprising 26 with their first episode of $\mathrm{CH}, 134$ with episodic $\mathrm{CH}, 10$ with chronic $\mathrm{CH}$, and 22 with probable $\mathrm{CH}$. Their age was $38.1 \pm 10.8$ years (mean \pm standard deviation) and 160 (83.3\%) of them were male. The age at the onset of $\mathrm{CH}$ was $29.6 \pm 12.5$ years and the $\mathrm{CH}$ duration was $8.4 \pm 8.1$ years. The score on the numeric rating scale for $\mathrm{CH}$ severity from the latest ictal period was $8.9 \pm 1.2$. The frequency of $\mathrm{CH}$ was $2.2 \pm$ 1.8 per day during the latest ictal period, and the total number of $\mathrm{CH}$ bouts was $8.7 \pm 13.2$.

Thirty (15.6\%) of the 192 patients with $\mathrm{CH}$ had comorbid migraine, comprising 18 with migraine without aura, 1 with migraine with aura, 3 with chronic migraine, and 8 with probable migraine. The $\mathrm{CH}$ duration was shorter in patients with $\mathrm{CH}$ with comorbid migraine than in those without (5.4 \pm 7.4 vs. 9.0 \pm 8.2 years, $p=0.008$ ), and the time delay to a $\mathrm{CH}$ diagnosis was shorter in patients with $\mathrm{CH}$ and comorbid migraine than in those without migraine ( $3.2 \pm 5.5$ vs. $5.9 \pm 7.3$ years, $p=0.032$ ). Episodic $\mathrm{CH}$ was less common ( $50.0 \%$ vs. $73.5 \%$, $p=0.010)$ and chronic $\mathrm{CH}$ was more common (13.3\% vs. $3.7 \%, p=0.033$ ) in patients with $\mathrm{CH}$ and comorbid migraine than in those without migraine. There were no significant differences between the patients with and without comorbid migraine in the age at study inclusion (37.6 \pm 8.4 vs. $38.2 \pm 11.3$ years, $p=0.781$ ), age at $\mathrm{CH}$ onset ( $32.2 \pm 11.6$ vs. $29.2 \pm 12.7$ years, $p=0.232$ ), or total number of bouts ( $7.6 \pm 10.2$ vs. $8.9 \pm$ 13.7 years, $p=0.625$ ) (Table 1 ). The following characteristics of $\mathrm{CH}$ and its comorbidity also did not differ significantly between patients with and without migraine history: HIT-6 score (69.1 \pm 7.3 vs. $67.6 \pm 8.4, p=0.374)$, GAD-7 score $(8.8 \pm$ 6.4 vs. $7.2 \pm 5.5, p=0.163$ ), PHQ-9 score ( $8.9 \pm 7.7$ vs. $7.1 \pm 6.0$, $p=0.170)$, PSS- 4 score (6.6 \pm 3.2 vs. $6.7 \pm 3.0, p=0.972)$, and EQ-5D score ( $0.8 \pm 0.2$ vs. $0.9 \pm 0.2, p=0.166)$ (Table 2$)$.

\section{Detailed history of migraine and $\mathrm{CH}$}

Nineteen of the 30 patients with $\mathrm{CH}$ and comorbid migraine participated in detailed interviews. The age at the onset of migraine was $27.6 \pm 9.3$ years. The onset of migraine preceded the onset of $\mathrm{CH}$ by $6.5 \pm 4.9$ years in 12 patients, the onset of $\mathrm{CH}$ preceded the onset of migraine by $9.7 \pm 5.5$ years in 4 patients, and the onset of both conditions occurred in the same year in 3 patients. These patients distinguished their $\mathrm{CH}$ from migraine based on $3.5 \pm 1.7$ features (range $1-8$ features): trigeminal autonomic symptoms $(n=14)$, pulsating character $(n=8)$, severity $(n=7)$, location of pain $(n=7)$, timing of pain $(n=6)$, presence of nausea $(n=6)$, premonitory symptoms (just feeling) $(n=5)$, frequency $(n=5)$, phonophobia or photophobia $(n=4)$, and agitation $(n=4)$. As curative medications for migraine, 6 patients took triptans, 11 took nonsteroidal anti-inflammatory drugs, and 4 took acetaminophen, while 1 patient did not take any medication. Eight patients reported that the migraine-associated pain was endurable and wanted to defer medication use until the cluster period, three patients did not take any curative medication, and five patients took acetaminophen or acetaminophen-combi-

Table 1. Comparative analysis of demographics according to the history of preceding migraine

\begin{tabular}{|c|c|c|c|c|}
\hline & Total $(n=192)$ & With migraine $(n=30)$ & Without migraine $(n=162)$ & $p$ \\
\hline Sex, female & $32(16.7)$ & $9(30.0)$ & $23(15.5)$ & 0.062 \\
\hline Age, years & $38.1 \pm 10.8$ & $37.6 \pm 8.4$ & $38.2 \pm 11.3$ & 0.781 \\
\hline Body mass index, $\mathrm{kg} / \mathrm{m}^{2}$ & $24.0 \pm 3.1$ & $23.0 \pm 2.9$ & $24.2 \pm 3.2$ & 0.056 \\
\hline Duration of $\mathrm{CH}$, years & $8.4 \pm 8.1$ & $5.4 \pm 7.4$ & $9.0 \pm 8.2$ & 0.008 \\
\hline Diagnostic delay time for $\mathrm{CH}$, years & $5.5 \pm 7.1$ & $3.2 \pm 5.5$ & $5.9 \pm 7.3$ & 0.032 \\
\hline Age at onset of $\mathrm{CH}_{\text {, years }}$ & $29.6 \pm 12.5$ & $32.2 \pm 11.6$ & $29.2 \pm 12.7$ & 0.232 \\
\hline Total number of $\mathrm{CH}$ bouts & $8.7 \pm 13.2$ & $7.6 \pm 10.2$ & $8.9 \pm 13.7$ & 0.625 \\
\hline NRS score for $\mathrm{CH}$ severity & $8.9 \pm 1.2$ & $8.8 \pm 1.5$ & $9.0 \pm 1.2$ & 0.270 \\
\hline Frequency of $\mathrm{CH}$ per day & $2.2 \pm 1.8$ & $2.5 \pm 2.2$ & $2.2 \pm 1.8$ & 0.475 \\
\hline \multicolumn{5}{|l|}{ Classification of $\mathrm{CH}^{*}$} \\
\hline First episode of $\mathrm{CH}$ & $26(13.5)$ & $7(23.3)$ & $19(11.7)$ & 0.088 \\
\hline Episodic CH & $134(69.8)$ & $15(50.0)$ & $119(73.5)$ & 0.010 \\
\hline Chronic $\mathrm{CH}$ & $10(5.2)$ & $4(13.3)$ & $6(3.7)$ & 0.033 \\
\hline Probable $\mathrm{CH}$ & $22(11.5)$ & $4(13.3)$ & $18(11.1)$ & 0.726 \\
\hline
\end{tabular}

Data are $n(\%)$ or mean \pm standard-deviation values.

${ }^{*} p$ value in Fisher's exact test of 0.028 .

$\mathrm{CH}$ : cluster headache, NRS: numeric rating scale. 
Table 2. Comparative analysis of clinical variables according to the history of preceding migraine

\begin{tabular}{|c|c|c|c|c|}
\hline & Total $(n=192)$ & With migraine $(n=30)$ & Without migraine $(n=162)$ & $p$ \\
\hline Smoking history & & & & 0.064 \\
\hline Current smoker & $82(42.7)$ & $7(23.3)$ & $75(46.3)$ & \\
\hline Ex-smoker & $35(18.2)$ & $7(23.3)$ & $28(17.3)$ & \\
\hline Never smoked & 75 (39.1) & $16(53.3)$ & $59(36.4)$ & \\
\hline Alcohol intake, glasses per week & $10.3 \pm 15.9$ & $3.7 \pm 7.9$ & $11.4 \pm 16.7$ & 0.001 \\
\hline Headache Impact Test-6 score & $67.8 \pm 8.2$ & $69.1 \pm 7.3$ & $67.6 \pm 8.4$ & 0.374 \\
\hline General Anxiety Disorder-7 score & $7.4 \pm 5.6$ & $8.8 \pm 6.4$ & $7.2 \pm 5.5$ & 0.163 \\
\hline Patient Health Questionnaire-9 score & $7.3 \pm 6.2$ & $8.9 \pm 7.7$ & $7.1 \pm 6.0$ & 0.170 \\
\hline Short-Form Perceived Stress Scale-4 score & $6.6 \pm 3.0$ & $6.6 \pm 3.2$ & $6.7 \pm 3.0$ & 0.972 \\
\hline EQ-5D health utility score ${ }^{*}$ & $0.8 \pm 0.2$ & $0.8 \pm 0.2$ & $0.9 \pm 0.2$ & 0.166 \\
\hline Allodynia Symptoms Scale-12 score during cluster headache & $2.7 \pm 3.9$ & $3.7 \pm 4.6$ & $2.5 \pm 3.8$ & 0.168 \\
\hline
\end{tabular}

Data are $n(\%)$ or mean \pm standard-deviation values.

${ }^{*}$ Quality of life was assessed by the EQ-5D, three-level instrument and converted into the EQ-5D health utility score.

EQ-5D: Euro0ol five-dimension.

nation medications. Three patients took preventive medication for migraine: one took cinnarizine, one took topiramate and amitriptyline, and one took candesartan and amitriptyline. Two patients had a history of episodic tension-type headaches additional to $\mathrm{CH}$ and migraine.

\section{DISCUSSION}

The key findings of our study were as follows: First, 30 (15.6\%) of the 192 patients had comorbid migraine, and the frequency of chronic $\mathrm{CH}$ was higher in patients with comorbid migraine than in those without migraine ( $13.3 \%$ vs. $3.7 \%)$. Second, the clinical characteristics and psychiatric comorbidity did not differ according to the presence of comorbid migraine in patients with $\mathrm{CH}$. Third, comorbid migraine was associated with a shorter duration of $\mathrm{CH}$ and shorter delay in diagnosing $\mathrm{CH}$.

To our knowledge, this is the first study to prospectively investigate migraine comorbidity and its relationship with $\mathrm{CH}$ in patients with ICHD-3-compatible $\mathrm{CH}$. Migraine is reportedly present in about one-fourth of patients with $\mathrm{CH}$, but the additional diagnosis of migraine based on the ICHD-3 has not been evaluated in patients with $\mathrm{CH} .{ }^{6}$ In our study, the prevalence of migraine comorbidity was $15.6 \%$ in all $\mathrm{CH}$ patients, and as higher as $40 \%$ in patients with chronic $\mathrm{CH}$. Our study results are consistent with a Swedish study finding migraine in $15.6 \%$ of patients with episodic $\mathrm{CH}$ and $22.2 \%$ of patients with chronic $\mathrm{CH} .{ }^{12}$ Regarding the prevalence of chronic $\mathrm{CH}$ being higher with migraine comorbidity, the traditional hypothesis is that migraines may culminate into $\mathrm{CH}^{21}$ However, both $\mathrm{CH}$ and migraine were considered as different clinical entities, with either of them potentially leading to the other. ${ }^{11,22}$ Furthermore, considering chronicity, a central sensitization or biological correlation could result in a closer association between chronic $\mathrm{CH}$ and migraine. ${ }^{23,24}$

In our study we found that most of the clinical parameters and psychiatric comorbidities did not differ significantly according to the presence of migraine comorbidity, except for the disease duration and diagnostic delay time in patients with $\mathrm{CH}$. The trigeminovascular system, parasympathetic nerve fibers (trigeminal autonomic reflex), and the hypothalamus are three key structures associated with $\mathrm{CH}^{7}{ }^{7}$ Despite $\mathrm{CH}$ and migraine having been categorized as different diseases, as mentioned above, $\mathrm{CH}$ presents with headache-associated symptoms such as nausea, photophobia, and phonophobia that are similar to those appearing in migraine. Moreover, considering their disease mechanisms, hypothalamus involvement in migraine pathophysiology is also closely associated with triggering $\mathrm{CH}_{.}^{25}$ Psychosocial problems including stress levels and the daily quality of life of patients with $\mathrm{CH}$ have not previously been researched in the context of the history of migraine comorbidity. In our study, stress and psychiatric comorbidities did not differ with the presence of comorbid migraine. These results are in line with previous studies showing that the presence of comorbid migraine does not affect the pain intensity and clinical characteristics of $\mathrm{CH} ;{ }^{4,6}$ furthermore, our results additionally indicate that psychological comorbidities do not differ among patients with $\mathrm{CH}$ according to the presence or absence of comorbid migraine.

Contrary to the belief that migraine can cause a diagnostic delay in $\mathrm{CH}_{2}{ }^{26}$ we found that the headache duration and diagnostic delay time were shorter in patients with $\mathrm{CH}$ and comorbid migraine than in those without migraine comorbidity. Although the underlying mechanism remains unclear, this may be due to the onset of migraine occurring earlier than that of $\mathrm{CH}$, which was reported in two-thirds of patients interviewed in our study; these patients may therefore have focused more on their headache and sought medical advice, 
which may have facilitated the early diagnosis of $\mathrm{CH}$. In addition, our patients with both $\mathrm{CH}$ and migraine reported that they regarded their migraine headaches as less severe than $\mathrm{CH}$. This may have resulted in a difference between the medications they used for migraine or $\mathrm{CH}$.

Our research was subject to some limitations. First, although we screened a large number of patients with $\mathrm{CH}$ who were recruited from a multicenter study, only a relatively small number of patients with comorbid migraine participated in the interview substudy. Thus, our results might not be generalizable before more patients are investigated in a populationbased study. Second, although our study included more patients with migraine than in other studies, the number was still not sufficient for a more-detailed comparison that also considered the subtypes of migraine. Third, the relationship between $\mathrm{CH}$ and migraine was judged based on the medical records and history-taking, and not in a prospective follow-up.

In conclusion, this study found no significant differences in the characteristics of $\mathrm{CH}$ and its comorbidities according to the presence of comorbid migraine. However, the chronic $\mathrm{CH}$ subtype appeared more often with comorbid migraine, which may make the diagnosis of $\mathrm{CH}$ faster and easier. The correct management of both primary headache disorders requires clinicians to check for the history of preceding migraine or to follow up the occurrence of migraine during the diagnosis and management of $\mathrm{CH}$.

\section{Conflicts of Interest}

The authors have no potential conflicts of interest to disclose.

\section{Acknowledgements}

This project was supported by grant from the Basic Science Research Program through the National Research Foundation of Korea funded by the Ministry of Education (2018R1D1A1B07040959).

\section{REFERENCES}

1. No authors listed. Headache Classification Committee of the International Headache Society (IHS). The International Classification of Headache Disorders, 3rd edition. Cephalalgia 2018;38:1-211.

2. Ljubisavljevic S, Zidverc Trajkovic J. Cluster headache: pathophysiology, diagnosis and treatment. J Neurol 2018 Aug 17 [Epub]. Available from: https://doi.org/10.1007/s00415-018-9007-4.

3. Burish M. Cluster headache and other trigeminal autonomic cephalalgias. Continuum (Minneap Minn) 2018;24:1137-1156.

4. Gaul C, Christmann N, Schröder D, Weber R, Shanib H, Diener HC, et al. Differences in clinical characteristics and frequency of accompanying migraine features in episodic and chronic cluster headache. Cephalalgia 2012;32:571-577.

5. Taga A, Russo M, Manzoni GC, Torelli P. Cluster headache with accompanying migraine-like features: a possible clinical phenotype. Headache 2017;57:290-297.
6. Zidverc-Trajkovic J, Podgorac A, Radojicic A, Sternic N. Migraine-like accompanying features in patients with cluster headache. How important are they? Headache 2013;53:1464-1469.

7. Vollesen AL, Benemei S, Cortese F, Labastida-Ramírez A, Marchese F, Pellesi L, et al. Migraine and cluster headache-the common link. J Headache Pain 2018;19:89.

8. Láinez MJ, Guillamón E. Cluster headache and other TACs: pathophysiology and neurostimulation options. Headache 2017;57:327-335.

9. Vukovic Cvetkovic V, Jensen RH. Neurostimulation for the treatment of chronic migraine and cluster headache. Acta Neurol Scand 2019;139:4-17.

10. Kudrow L, Kudrow DB. Inheritance of cluster headache and its possible link to migraine. Headache 1994;34:400-407.

11. D’Amico D, Centonze V, Grazzi L, Leone M, Ricchetti G, Bussone G. Coexistence of migraine and cluster headache: report of 10 cases and possible pathogenetic implications. Headache 1997;37:21-25.

12. Steinberg A, Fourier C, Ran C, Waldenlind E, Sjöstrand C, Belin AC. Cluster headache-clinical pattern and a new severity scale in a Swedish cohort. Cephalalgia 2018;38:1286-1295.

13. Choi YJ, Kim BK, Chung PW, Lee MJ, Park JW, Chu MK, et al. Impact of cluster headache on employment status and job burden: a prospective cross-sectional multicenter study. J Headache Pain 2018;19:78.

14. Headache Classification Committee of the International Headache Society (IHS). The International Classification of Headache Disorders, 3rd edition (beta version). Cephalalgia 2013;33:629-808.

15. Song TJ, Kim YJ, Kim BK, Kim BS, Kim JM, Kim SK, et al. Characteristics of elderly-onset ( $\geq 65$ years) headache diagnosed using the International Classification of Headache Disorders, third edition beta version. J Clin Neurol 2016;12:419-425.

16. Sohn JH, Choi YJ, Kim BK, Chung PW, Lee MJ, Chu MK, et al. Clinical features of probable cluster headache: a prospective, cross-sectional multicenter study. Front Neurol 2018;9:908.

17. Seo JG, Park SP. Validation of the Generalized Anxiety Disorder-7 (GAD-7) and GAD-2 in patients with migraine. J Headache Pain 2015; 16:97.

18. Seo JG, Park SP. Validation of the Patient Health Questionnaire-9 (PHQ9) and PHQ-2 in patients with migraine. J Headache Pain 2015;16:65.

19. Lee EH, Chung BY, Suh CH, Jung JY. Korean versions of the Perceived Stress Scale (PSS-14, 10 and 4): psychometric evaluation in patients with chronic disease. Scand J Caring Sci 2015;29:183-192.

20. Lee YK, Nam HS, Chuang LH, Kim KY, Yang HK, Kwon IS, et al. South Korean time trade-off values for EQ-5D health states: modeling with observed values for 101 health states. Value Health 2009;12:11871193.

21. Wei DY, Yuan Ong JJ, Goadsby PJ. Cluster headache: epidemiology, pathophysiology, clinical features, and diagnosis. Ann Indian Acad Neurol 2018;21:S3-S8

22. Boes CJ, Capobianco DJ, Matharu MS, Goadsby PJ. Wilfred Harris' early description of cluster headache. Cephalalgia 2002;22:320-326.

23. Mosnaim AD, Puente J, Wolf ME. Biological correlates of migraine and cluster headaches: an overview of their potential use in diagnosis and treatment. Pragmat Obs Res 2010;1:25-32.

24. D’Andrea G, Terrazzino S, Leon A, Fortin D, Perini F, Granella F, et al. Elevated levels of circulating trace amines in primary headaches. Neurology 2004;62:1701-1705.

25. Overeem S, Van Vliet JA, Lammers GJ, Zitman FG, Swaab DF, Ferrari MD. The hypothalamus in episodic brain disorders. Lancet Neurol 2002;1:437-444

26. Bahra A, Goadsby PJ. Diagnostic delays and mis-management in cluster headache. Acta Neurol Scand 2004;109:175-179. 